\title{
pH dependence of the protein orientation in self-assembled bacteriorhodopsin/polycation multilayer films
}

\author{
Tapio Jussila, ${ }^{1}$ Meiling Li, ${ }^{2}$ Nikolai V. Tkachenko, ${ }^{1}$ Sinikka Parkkinen, ${ }^{3}$ \\ Baofang Li, ${ }^{2}$ Long Jiang, ${ }^{2}$ and Helge Lemmetyinen ${ }^{1}$ \\ ${ }^{1}$ Institute of Materials Chemistry, Tampere University of Technology, P.O. Box 541, 33101 Tampere \\ ${ }^{2}$ Laboratory of Colloid and Interface Science, Center for Molecular Science, Institute of Chemistry, \\ Chinese Academy of Sciences, Beijing, 100101 People's Republic of China \\ ${ }^{3}$ University of Joensuu, Department of Biology, P.O. Box 111, 80101, Finland
}

\begin{abstract}
Bacteriorhodopsin (bR) multilayer films were successfully prepared by alternating depositions of cationic poly(diallyldimethylammonium chloride) (PDAC) polyelectrolyte and purple membrane (PM). The advantage of the method when compared to other thin film techniques is its simplicity to prepare large selfassembled films. The orientations of the bR molecules in the films were studied by a transient photovoltage method. The polarity of the photoresponse signals showed that in basic conditions the cytoplasmic side of PM was adsorbed onto the polyelectrolyte layer, whereas in acidic conditions the extracellular side was adsorbed onto the polyelectrolyte layer. The photocycle of the bR in the films was studied by using the conventional flash photolysis method. The photochemical behavior of bR in dry polycationic films was similar to that in other types of dry films and the intermediate lifetimes were almost independent of the protein orientation.

The photovoltage signals introduce opposite proton transport directions for the formation of the $\mathrm{K}$ intermediate of the photocycle and for the successive relaxation to $\mathrm{M}$ intermediate. The charge displacement associated to the M intermediate was several times greater than that of the K intermediate. The fittings of the transient photovoltage signals resulted the values of 2, 15, and 70 microseconds for the time constants of the 3-exponential formation of the $\mathrm{M}$ intermediate. The kinetic data were compared to the results obtained with the transient absorption measurements.
\end{abstract}

\section{INTRODUCTION}

Bacteriorhodopsin (bR), a transmembrane protein in the purple membrane (PM) of Halobacterium salinarium, functions as a light driven proton pump. The largescale applications to convert sunlight into chemical or electrical energy has not been successful, but as a very stable substance it is applied for different types of optoelectrical devices, e.g., in photochromic memories, image processing, and holographic applications [1, 2].

Several kinds of techniques have been introduced to prepare anisotropic bR samples. A permanent orientation of the bR molecules in gel or polymer matrices has been achieved by accomplishing the solidification under an electric field $[3,4]$. To produce self-assembled films, other techniques, including an immobilizing PM onto a solid support with a chemisorption, an electrical sedimentation, and by using antibody techniques, have been used [4]. The conventional Langmuir-Blodgett (LB) technique has also been applied to prepare oriented PM multilayer films layer by layer $[4,5,6,7,8]$.

Due to acidic end groups of the lipids and the exterior segments of bR protein, PM has a net negative surface charge on both sides of the membrane. The difference in the surface charge densities in the extracellular (EC) and cytoplasmic (CP) sides produce a permanent dipole moment over the membrane, which exposes the orientation of the bR molecules [9]. In the uniformly oriented PM the charge displacement coupled to the events of the photocycle produces an observable photoelectrical signal offering a method for the study of the proton pumping mechanism and the basis for the technical applications of the bR films as well.

An alternate electrostatic deposition of anionic and cationic polyelectrolytes is a recent method to prepare self-assembled multilayer films [10]. Thanks to the negatively charged surfaces the multilayer structure of PM can be achieved by the alternate depositions of cationic polyelectrolyte and PM onto a solid substrate $[4,11,12,13,14]$. The surface charge densities depend on the $\mathrm{pH}$, which opens the possibility of controlling the orientation of the $\mathrm{bR}$ molecules on the polyelectrolyte layer. In the present study the correlation between the orientation of bR in (PDAC/PM $)_{n}$ multilayer films ( $\mathrm{n}$ is the number of dippings) and the $\mathrm{pH}$ of the PM suspension was studied. The orientation of the bR molecules was concluded from the direction of the photo-induced proton pumping, which was determined by the photovoltage method.

\section{MATERIALS AND METHODS}

The deposition of the polyelectrolyte/PM bilayer was the follows [11]: 1) dipping the solid quartz substrate in a solution of polyelectrolyte for $5 \mathrm{~min}$, 2) rinsing the polyelectrolyte covered substrate with Milli-Q water for $2 \mathrm{~min}$ and drying under the nitrogen flow for $10 \mathrm{~min}$, 3) dipping the covered substrate in a suspension of 
PM for $3 \mathrm{~min}$, 4) rinsing the polyelectrolyte/PM covered substrate with Milli-Q water ( $\mathrm{pH}$ adjusted to the same value as that of the PM suspension) for 2 min and drying under the nitrogen flow for $10 \mathrm{~min}$. The maximum numbers of the membrane layers were 30 and 90 for the electrical and optical measurements, respectively. All the dippings were done at room temperature.

Halobacterium salinarium wild type was grown and the purple membrane isolated according to the method described by Oesterhelt and Stoeckenius [15]. The concentrations of the PM suspensions were $0.5 \mathrm{mg} / \mathrm{ml}$, the optical densities (OD) 0.9 at $570 \mathrm{~nm}$, and the absorption ratio of $\mathrm{A}_{280} / \mathrm{A}_{570} 2.2 \quad\left(\mathrm{~A}_{280}\right.$ and $\mathrm{A}_{570}$ represent the absorptions of the aromatic amino acid residues in the $\mathrm{bR}$ protein, and the absorption of the protonated Schiff base retinal, respectively). The $\mathrm{pH}$ 3-11 was adjusted with $\mathrm{H}_{3} \mathrm{BO}_{3} / \mathrm{NaOH}$ or Tris buffers. The concentration of the positive polyelectrolyte poly(diallyldimethylammonium chloride) (PDAC, Aldrich Chemical Company, medium molecular weight, average $\mathrm{M}_{\mathrm{W}} \sim 200,000-350,000,20$ wt. \% in water) was $2 \mathrm{mg} / \mathrm{ml}$ in a $0.5 \mathrm{M} \mathrm{NaCl}$ solution. The $\mathrm{pH}$ of the solution was adjusted to 6.8 with $\mathrm{KH}_{2} \mathrm{PO}_{4} / \mathrm{NaOH}$ buffer. All the solutions were made in Milli-Q water.

Quartz plates were used as solid substrates. Before any depositions the clean plates were sonicated in a $\mathrm{KOH}$ solution followed by rinsing in Milli-Q water to obtain negatively charged surfaces capable for the PDAC absorption. The absorption spectra of the PDAC/PM films were measured with a Shimadzu UV-2501PC spectrophotometer and the transient absorption using a nanosecond flash photolysis method. The samples were excited by the second harmonic of the Nd:YAG laser: wavelength $532 \mathrm{~nm}$, pulse duration $10 \mathrm{~ns}$ and energy per pulse $c a$. $2 \mathrm{~mJ}$. A halogen lamp with a constant emission coupled with a monochromator was used as the monitoring light source. Several tens of measurements were averaged to improve the signal-to-noise ratio. For controlling the experiments the purple membrane was substituted by the retinal free membrane, apo-membrane (AM), which is a bleaching product of the PM and unable to the proton pumping [16]. The $\mathrm{pH}$ of the AM suspension was adjusted to 7 with Tris buffers and the depositions were accomplished with the same procedure as for PM.

Quartz plates covered with a conductive semitransparent indium-tin oxide (ITO) film were used as substrates for the films deposited for electrical measurements. If the film consists of only a few layers, the possible holes in the probed film on ITO plate may cause short circuits between the electrodes. Therefore in the films where only a few layers of PM were deposited, several AM layers were deposited before PM to obtain an insulating layer needed for the photovoltage measurments.

The instrument for the photovoltage measurements is described elsewhere [6]. The ITO layer was used as the first electrode and an InGa low-melting alloy (liquid metal at room temperature) as the second electrode.
The input resistances of the preamplifier were $100 \mathrm{M} \Omega$, 10 or $100 \mathrm{G} \Omega$, which correspond to $\mathrm{ca}$. $5 \mathrm{~ms}, 0.5 \mathrm{~s}$ or $5 \mathrm{~s}$ relaxation time constants of the measuring system, respectively. The ITO electrode was used as a ground electrode, therefore the photovoltage increases when a positive net charge moves from the ITO to the drop electrode side. The Nd:YAG laser, the same as in the transient absorption measurements was used as the excitation source. For high responses saturation of the preamplifier was prevented by reducing the intensity of the excitation light with neutral density filters. The average of only a few measurements were needed to achieve a high signal-to-noise ratio. The optical and electrical measurements were done at a relative humidity of approximately $35 \%$ and a temperature of $20^{\circ} \mathrm{C}$.

The transient data were fitted to a sum of exponential functions using a non-linear least squares method

$$
f(t)=\sum_{i=1}^{n} \mathrm{a}_{i} e^{-t / \tau_{i}}
$$

where $\mathrm{a}_{i}$ is the amplitude associated to the corresponding time constant $\tau_{i}$.

\section{RESULTS AND DISCUSSION}

3.1. Absorption spectra of multilayer films. Since the PDAC polyelectrolyte has no absorption in the 250$800 \mathrm{~nm}$ wavelength range, the deposition of PM layer by layer can be monitored spectroscopically. The absorption spectrum of the PM suspension and the spectra of $(\mathrm{PDAC} / \mathrm{PM})_{\mathrm{n}}$ multilayer films up to 60 layers deposited from the suspension at pH 5.0 are presented in Figure 1. The absorption bands of (PDAC/PM) n films are broadened and the maxima are shifted to the blue. The optical density at $560 \mathrm{~nm}$ increases linearly to the value of 0.3 with 60 dippings (Figure 1, inset). The same value of the absorption is reached with only 90 dippings for the film deposited at pH 8 (Figure 1, inset). The reason for the higher adsorption at pH 5 might be in the decreased hydroxide ion concentration on the surface of the polyelectrolyte layer.

After subtracting the scattered light the absorbances for a monolayer (on one side of the plate only) were $1.56 \times 10^{-3}(\mathrm{pH} 5)$ and $1.19 \times 10^{-3}(\mathrm{pH})$. The unit cell size of the two-dimensional hexagonal lattice of the bR molecules in the PM is $63 \AA$, and the absorption cross section $2.4 \AA$ [5]. Thus the absorbance of the PM monolayer can be expected to be $1.2 \times 10^{-3}$. The measured values are close to the calculated one, which suggests the absorption of approximately an equal amount of PM required for the monomolecular depositions. The values are also close to those reported earlier for the $(\mathrm{PDAC} / \mathrm{PM})_{\mathrm{n}}$ film [11], but several times higher than those for the bR/soya-PC LB films [5]. The OD of the control sample (PDAC/AM) 10 presents a smaller deposition for the $\mathrm{AM}$ than for the $\mathrm{PM}$ in the corresponding (PDAC/PM) 10 film (Figure 1). The reason might be the changed surface charge densities of the retinal free membrane [17]. 


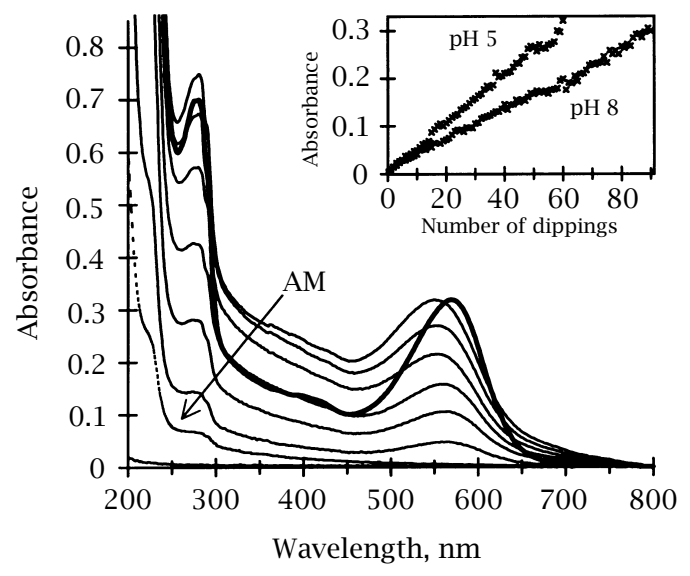

Figure 1. Absorption spectra of the PM suspension (thick line, normalized at $\sim 560 \mathrm{~nm})$, and the $(P D A C / P M)_{\mathrm{n}}$ (thin lines) and the (PDAC/AM) 10 (dashed line) multilayer films. The numbers ( $n$ ) of the PM layers (on both sides of the plate) are 0,10,20,30,40,50, and 60. The $\mathrm{pH}$ of the suspensions of $P M$ and $A M$ were 5 and 7 , respectively. The inset represents the maximum absorbance at $\sim 560 \mathrm{~nm}$ of the measured spectra after each PM dipping at $\mathrm{pH} 5$ and $\mathrm{pH} 8$.

3.2. Transient photovoltage of multilayer films. The proton movement takes place in the direction of the extracellular side during the formation of the $\mathrm{M}$ intermediate in microsecond time domain $[18,19]$. In the capacitative photovoltage system the electric response corresponds to the vectorial charge transfer in the direction perpendicular to the film surface. The opposite polarities of the photovoltage signals in Figure 2 display opposite orientations of the bR molecules in the $(\mathrm{PDAC} / \mathrm{PM})_{\mathrm{n}}$ films deposited from the basic or acidic suspensions (curves 1 and 2, respectively). The adsorption of the PM depends on the charge densities of the anionic surfaces of the membrane. In neutral and basic solutions the $\mathrm{CP}$ surface is more negative than the EC surface $[20,21]$ and the permanent dipole moment is directed from the $\mathrm{CP}$ side toward the EC side. Thus, as was expected, under the basic conditions the PM were adsorbed with the $\mathrm{CP}$ side onto the positive polyelectrolyte layer (curve 1 in Figure 2). In neutral suspension it is not, however, completely obvious as to which side the membrane absorbs onto the polyelectrolyte layer, since the $\mathrm{CP}$ side of the membrane is more hydrophilic than the EC side [18]. In consequence of the protonation of some amino acid residues and lipid head groups the surface charge densities are changed in the acidic suspension and the direction of the permanent dipole moment is converted toward the CP side [9]. Due to the changed surface charge densities the membrane is adsorbed with the EC side onto the polyelectrolyte layer (curve 2 in Figure 2). In the pH range of 6-7 the polarities of the measured signals varied depending on the position of the electrode on the samples. This indicates that the adsorption of the PM takes place randomly with both surfaces of the membrane fragments.

A microsecond timescale photovoltage signal is generated from a fast charge movement toward one elec- trode followed by a slower movement toward another electrode (Figure 2). The amplitudes of the components of the signal correspond to the translocation distances of the net charge $[18,19]$. In addation to the proton transport the movements of the charged amino acid side chains might also have an influence on the photovoltage signal. The shape of the measured photovoltage signals of the (PDAC/PM) multilayer film is in accordance with the results reported for other oriented bR systems [6, 18, 22, 23]. The fast photoresponse component is associated with the protonated Schiff base retinal isomerization from the all-trans to the 13-cis configuration in a picosecond and the successive relaxation to the $\mathrm{K}$ intermediate. The negative charge displacement of the $\mathrm{K}$ intermediate indicates the reversed charge movement at the beginning of the photocycle compared to the net proton pumping direction (the positive direction corresponds to the native proton pumping direction from the $\mathrm{CP}$ to the EC side of the membrane) $[18,19]$. The subsequent phase in the microsecond timescale is attributed to the M state formation. The charge displacement distance for the $\mathrm{M}$ state is four times longer than for the $\mathrm{K}$ state [3, 19], which correlates well to the value of $\sim 0.3$ obtained from the amplitudes of the opposite components of the curves in Figure 2.

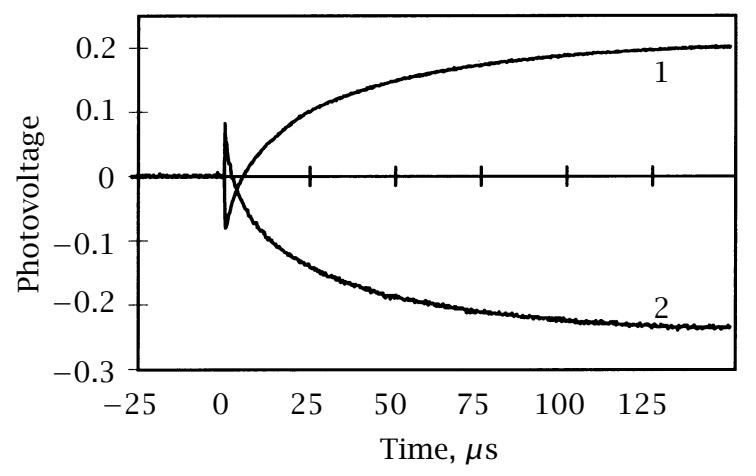

Figure 2. Transient photovoltages of self-assembled $(P D A C / P M)_{30}$ multilayer films. The $\mathrm{pH}$ of the PM suspensions were 9 (curve 1) and 5 (curve 2). The signals are an average of several measurements with $100 \mathrm{~s}$ delay times. The relaxation time constant of the measuring system was $4 \mathrm{~ms}$.

The typical time constants obtained from 2exponential fittings of the photovoltage signals are 8 and $50 \mu \mathrm{s}$ for the formation of the $\mathrm{M}$ intermediate in the $(\mathrm{PDAC} / \mathrm{PM})_{\mathrm{n}}$ films, the amplitude of the latter being 1-2 times larger. The lifetimes agree well with the 2-exponential formation of the $\mathrm{M}$ state in the dry LB films [6]. The 3-exponential fitting improved the fit remarkably giving time constants of 2,15 , and $70 \mu \mathrm{s}$. The shortest corresponds to the lifetime of the K intermediate of bR in the suspension. The charge displacement for the K-to-L transition has been reported to be very short and opposite to the proton pumping direction $[18,19]$. For the $(\mathrm{PDAC} / \mathrm{PM})_{\mathrm{n}}$ films the amplitudes of the 
three exponentials indicate that the net charge movement takes place in the direction of the proton pumping during the K-M sequence of the photocycle. The relative amplitudes of the $2 \mu \mathrm{s}$ component are rather high, $10-30 \%$ of the total signal amplitude.

3.3. Intensities of the signals. In the capacitative photovoltage system the diameter of the drop electrode is much larger than the distance between electrodes, and thus the induced photovoltage signal is independent of the probed area [6]. For the homogeneous layer-by-layer deposition the amplitude of the photovoltage signal is linearly proportional to the number of PM layers. Figure 3 presents the intensities of the photovoltage $150 \mu \mathrm{s}$ after the excitation as a function of the number of deposited PM layers. Before the deposition of the PDAC/PM bilayers four PDAC/AM bilayers were deposited to form the insulation film needed for the high resistance film. The photovoltage is a linear function of the number of the deposited layers after the first dipping.

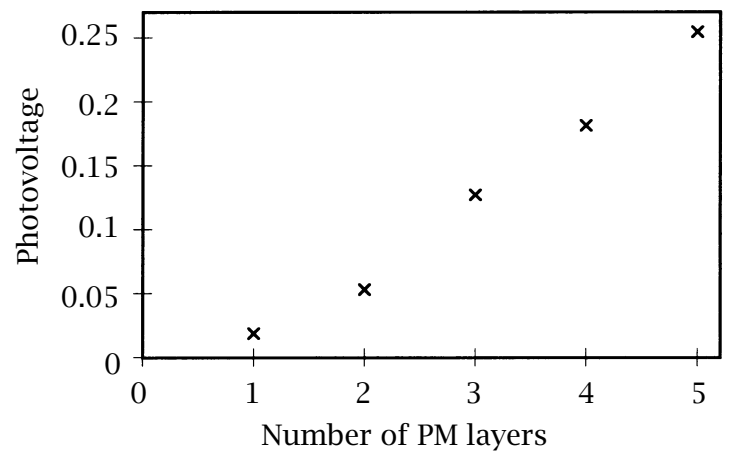

Figure 3. Photovoltage of the $(P D A C / P M)_{\mathrm{n}}$ film as a function of the number of the layers $(n=1-5)$ observed $150 \mu \mathrm{s}$ after the flash excitation. The photovoltages were measured after the subsequent PDAC depositions. The $\mathrm{pH}$ of the PM suspension was 5 .

The intensities of the photoresponses of the $(\mathrm{PDAC} / \mathrm{PM})_{\mathrm{n}}$ films are 10-100 fold higher than those achieved for bR in LB films (1.2 $\mathrm{mV}$ for the M intermediate in bR monolayer [6]). There are a few reasons for this. Firstly, the concentration of the bR monomers in LB films is lowered from the natural value of $3: 1$ of the bR:lipids weight ratio in PM, to the value of 2:1 of bR:soya-PC [5]. Secondly, the voltage is inversely proportional to the distance of the electrodes. The insulation layers in the LB films consisted of twenty stearic acid layers, which increased the film thickness of $50 \AA$ of the PM to the value of $1100 \AA$ of the LB film [9], while in the $(\mathrm{PDAC} / \mathrm{PM})_{\mathrm{n}}$ film the thickness of the PDAC layer is only $10 \%$ of the whole film thickness [11]. Thirdly, the bR:soya-PC films contained small holes [24]. With spectroscopic measurements only the first and third point are significant. The absorbance per deposited layer of bR:soya-PC is $0.455 \times 10^{-3}$ [5], which is only $30-40 \%$ of the absorbance of the PDAC/PM layer. The much higher absorbance of the (PDAC/PM) films (especially at $\mathrm{pH} 5$ ) suggests a denser film formation and perhaps excess adsorption of PM. A PM patch is large, having a diameter 200 times greater than the thickness $(5 \mathrm{~nm})$, and therefore its ability to form films with a regular brick wall structure might be incomplete and the overlapping of the membrane edges might be possible.

3.4. Transient absorption. The flash spectroscopy measurements were carried out to verify that the origin of the photoresponse is the proton transport during the formation of the $\mathrm{M}$ state. The photovoltage measurements display similar kinetics as the transient absorptions of the formation of the M state at $410 \mathrm{~nm}$ in the films deposited in the PM suspension of $\mathrm{pH} 5$ or 8 . The 2-exponential global fittings of the decay curves at 410 and $620 \mathrm{~nm}$ (Figure 4) result in time constants of 8 and $54 \mu$ s fot the formation of the M intermediate. The wavelength of $620 \mathrm{~nm}$ represents the absorption of the $\mathrm{K}_{590}$ intermediate and also partly the $\mathrm{ab}$ sorption of the successive $\mathrm{L}_{550}$ intermediate of the photocycle of bR. The obtained time constants correlate well with the values achieved with photovoltage measurements. Due to the low signal-to-noise ratio, and the fast component of the relaxation of the photomultiplier induces by the scattered laser light, the determination of the $1-2 \mu$ s component is not feasible.

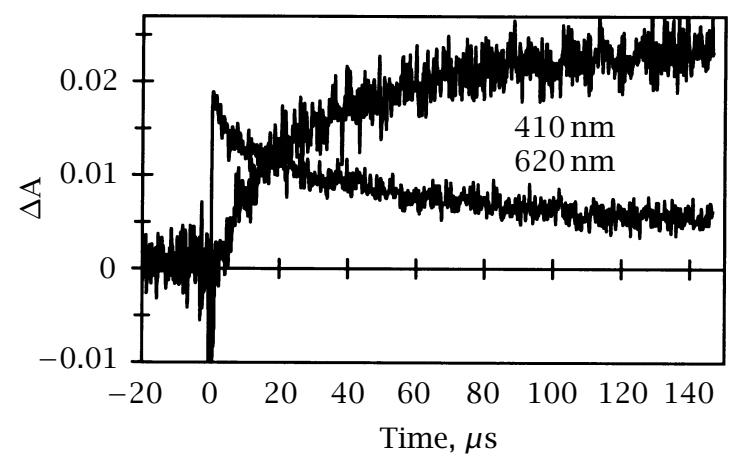

Figure 4. Transient absorption of the (PDAC/PM) 60 multilayer film. The $\mathrm{pH}$ of the PM suspension was 5. The monitoring wavelength were 410 and $620 \mathrm{~nm}$.

\section{CONCLUSIONS}

The alternating layer-by-layer deposition of the polyelectrolyte and PM is a very simple technique to produce oriented multilayer films avoiding extra bulky matrix materials. The photoexcitation of the bR protein in the high resistance $(\mathrm{PDAC} / \mathrm{PM})_{\mathrm{n}}$ film induce a proton movement to the cytoplasmic side of the bR protein during the fast formation of the $\mathrm{K}$ intermediate. During the relaxation to the $\mathrm{M}$ intermediate the proton moves in the direction of the proton pumping to the extracellular side. The photovoltage response displays opposite orientations for the bR proteins in the films deposited from the acidic or basic PM suspensions. In the basic and neutral suspensions the adsorption of the PM onto the cationic PDAC polyelectrolyte layer takes place with the cytoplasmic side of the membrane, but 
in the acidic suspensions with the extracellular side of the membrane. The kinetics with time constants of 2 , 15 , and $70 \mu \mathrm{s}$ for the formation of the $\mathrm{M}$ intermediate is independent of the $\mathrm{pH}$ on the PM deposition.

\section{ACKNOWLEDGEMENTS}

The work was financially supported by The Academy of Finland.

\section{REFERENCES}

[1] R. R. Birge and R. B. Gross, An Introduction to Molecular Electronics, M. C. Petty, M. R. Bryce, and D. Bloor (Ed.), Arnold, London, 1995, 315.

[2] N. Hampp, Chem. Rev. 100 (2000), 1755.

[3] A. Dér, L. Oroszi, Á. Kulcsár, L. Zimányi, R. TóthBoconádi, L. Keszthelyi, W. Stoeckenius, and G. Váró, Proc. Natl. Acad. Sci. USA 96 (1999), 2776.

[4] J.-A. He, L. Samuelson, L. Li, J. Kumar, and S. K. Tripathy, Adv. Mater. 11 (1999), 435.

[5] M. Ikonen, J. Peltonen, E. Vuorimaa, and H. Lemmetyinen, Thin Solid Films 213 (1992), 277.

[6] M. Ikonen, A. Sharonov, N. Tkachenko, and H. Lemmetyinen, Adv. Mater. Opt. Electr. 2 (1993), 115.

[7] M. Ikonen, A. Y. Sharonov, N. Tkachenko, and H. Lemmetyinen, Adv. Mater. Opt. Electr. 2 (1993), 211.

[8] H. Lemmetyinen and M. Ikonen, Trends Photochem. Photobiol. 3 (1994), 413.

[9] K. Barabás, A. Dér, Zs. Dancsházy, P. Ormos, and L. Keszhelyi, Biophys. J. 43 (1983), 5.
[10] G. Decher, J. D. Hong, and J. Schmitt, Thin Solid Films 210/211 (1992), 831.

[11] J.-A. He, L. Samuelson, L. Li, J. Kumar, and S. K. Tripathy, Langmuir 14 (1998), 1674.

[12] J.-A. He, L. Samuelson, L. Li, J. Kumar, and S. K. Tripathy, J. Phys. Chem. B. 102 (1998), 7067.

[13] M. Li, B. Li, L. Jiang, T. Jussila, N. Tkachenko, and H. Lemmetyinen, Langmuir 16 (2000), 5503.

[14] T. Jussila, M. Li, N. V. Tkachenko, S. Parkkinen, and H. Lemmetyinen, in Recent Research Developmentes in Photochemistry \& Photobiology in press (2000).

[15] D. Oesterhelt and W. Stoeckenius, Methods. Enzymol. 31 (1974), 667.

[16] D. Oesterhelt, Methods. Enzymol. 88 (1982), 10.

[17] S. Checover, E. Nachliel, N. A. Dencher, and M. Gutman, Biochemistry 36 (1997), 13919.

[18] H.-W. Trissl, Photochem. Photobiol. 51 (1990), 793.

[19] L. Keszthelyi and P. Ormos, J. Membr. Biol. 109 (1989), 193.

[20] E. Nachliel, M. Gutman, S. Kiryati, and N. A. Dencher, Proc. Natl. Acad. Sci. USA 93 (1996), 10747.

[21] U. Alexiev, T. Marti, M. P. Heyn, H. G. Khorana, and P. Scherrer, Biochemistry 33 (1994), 298.

[22] L. A. Drachev, A. D. Kaulen, and V. P. Skulachev, FEBS Lett. 87 (1978), 161.

[23] H.-W. Trissl, Biochem. Biophys. Acta 723 (1983), 327.

[24] H. E.-M. Niemi, M. Ikonen, M. Levlin, and H. Lemmetyinen, Langmuir 9 (1993), 2436. 


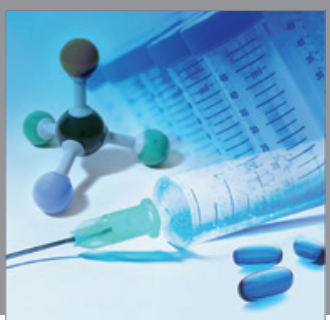

International Journal of

Medicinal Chemistry

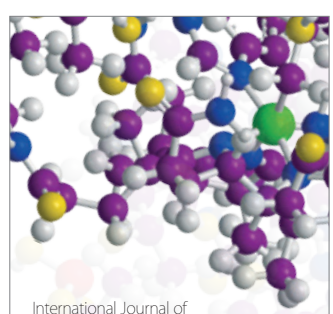

Carbohydrate Chemistry

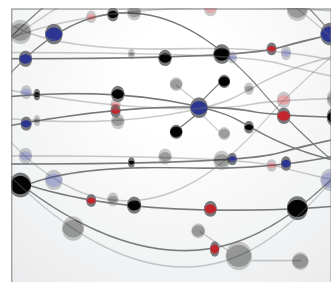

The Scientific World Journal
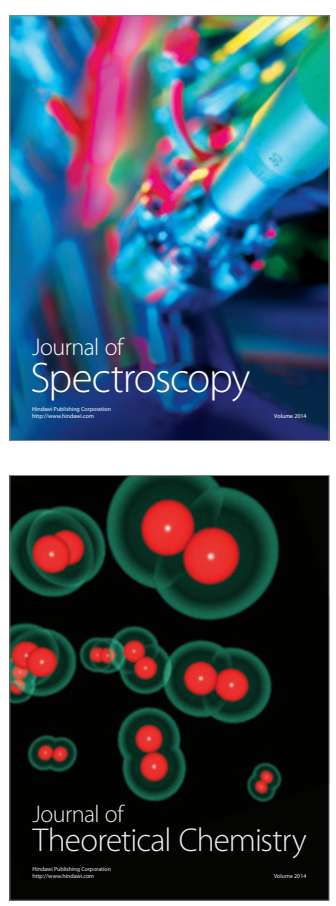
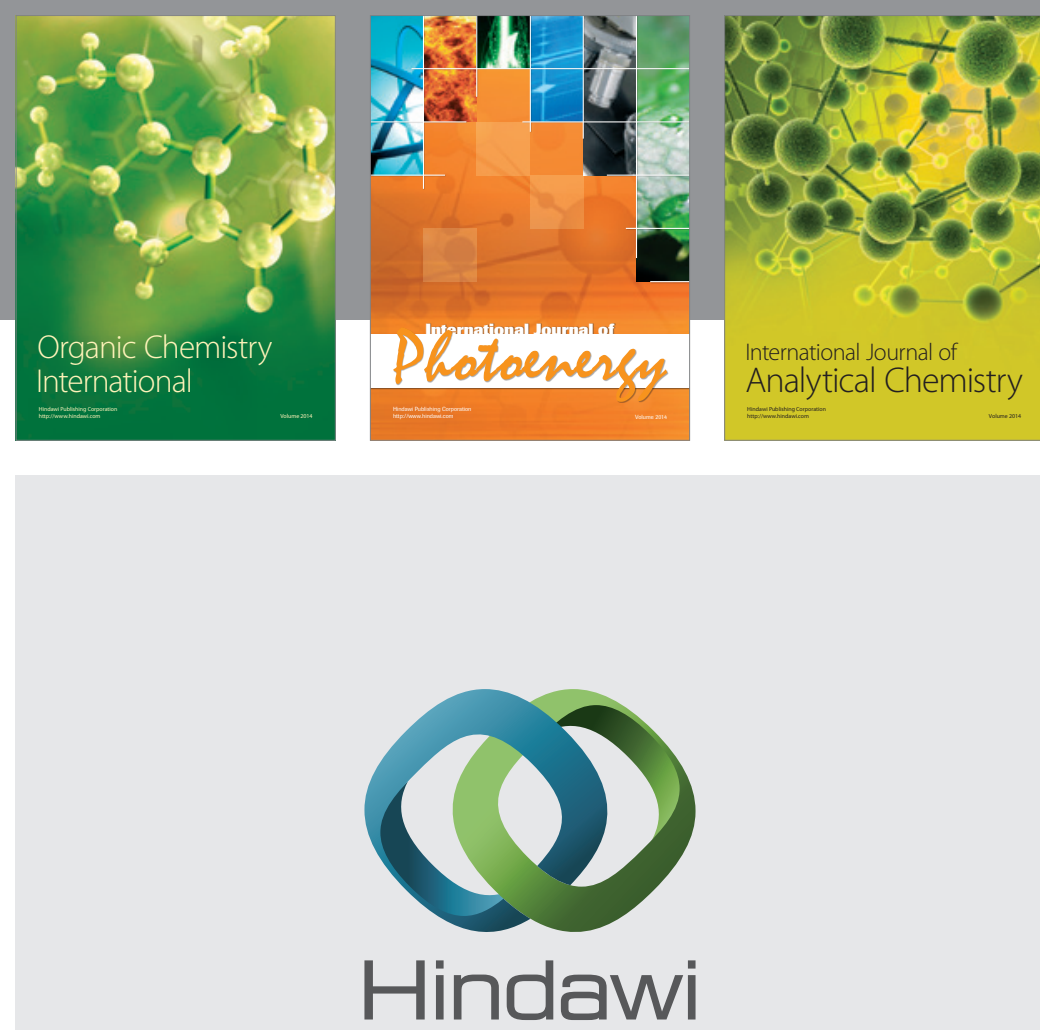

Submit your manuscripts at

http://www.hindawi.com
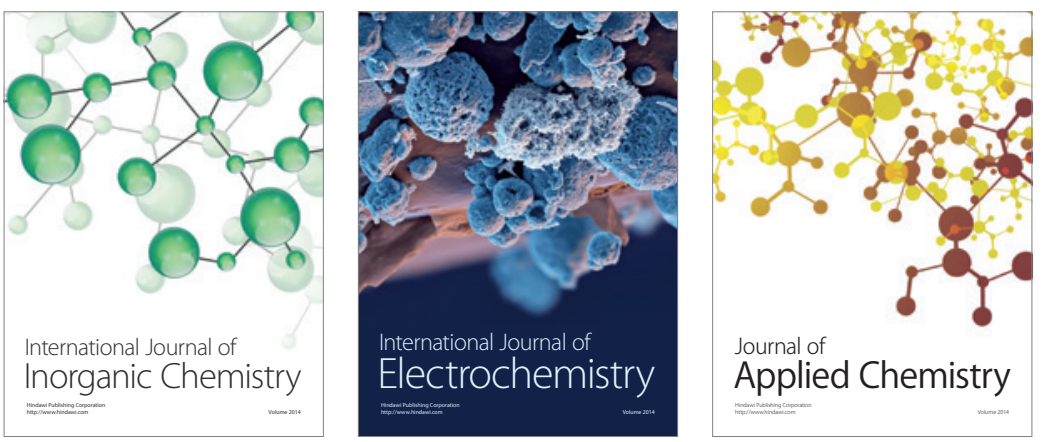

Journal of

Applied Chemistry
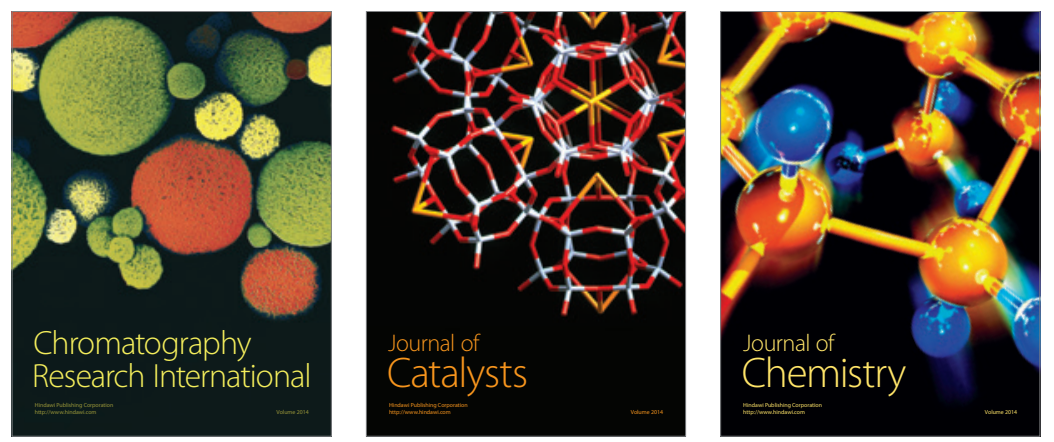
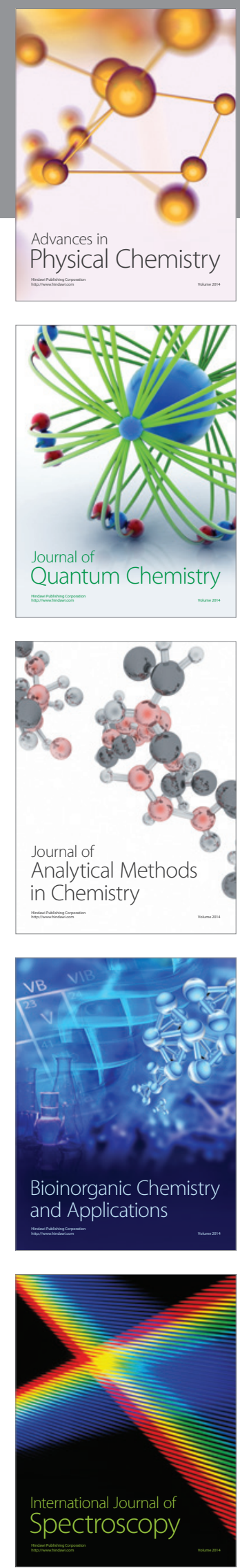